\title{
Anabolic steroid induced hypogonadism treated with human chorionic gonadotropin
}

\author{
GV Gill
}

\begin{abstract}
Summary
A case is presented of a young competitive body-builder who abused anabolic steroid drugs and developed profound symptomatic hypogonadotrophic hypogonadism. With the help of prescribed testosterone (Sustanon) he stopped taking anabolic drugs, and later stopped Sustanon also. Hypogonadism returned, but was successfully treated with weekly injections of human chorionic gonadotropin for three months. Testicular function remained normal thereafter on no treatment. The use of human chorionic gonadotropin should be considered in prolonged hypogonadotrophic hypogonadism due to anabolic steroid abuse.
\end{abstract}

Keywords: anabolic steroids; drug abuse; hypogonadism; human chorionic gonadotropin

The misuse of anabolic steroid drugs to enhance physique, body strength and athletic performance is well-known. ${ }^{1-5}$ High doses and/or multiple drug regimes are often adopted, ${ }^{15}$ leading to potentially serious sideeffects. These include suppression of endogenous secretion of follicle-stimulating hormone (FSH) and luteinising hormone (LH), leading to hypogonadotrophic hypogonadism (often manifesting itself as reduced testicular size, azoospermia or impotence). This may be difficult to treat and can persist for some time even after cessation of anabolic steroid abuse. A case is presented of an anabolic steroid abusing body-builder with this problem who was treated initially with prescribed testosterone injections (Sustanon) to encourage his withdrawal from illicit anabolic drugs; and later with human chorionic gonadotropin (HCG) to restore potency.

\section{Case report}

A 17-year-old man noted a right testicular swelling, and a varicocoele was found when he sought medical advice. When admitted for surgical excision he complained of erectile impotence, and scant body hair was noted. A testicular biopsy was performed at operation, which revealed histologically severely reduced spermatotogenesis. He was referred to an Endocrine Unit. His puberty had been normal and the impotence was of about four months duration. He also complained of sore nipples, low libido, and reduced body hair (he had shaved only once in four months). He was a competitive body-builder and admitted to anabolic steroid use for at least six months. $\mathrm{He}$ did not relate this to his current problems however, as he felt he had been taking 'safe anabolics'. It was difficult to obtain an accurate drug history, but he had taken nandralone, Sustanon, and possibly stanazolol. When he could, he took danazol to counteract nipple tenderness.

Examination revealed a muscular and fitlooking man with mild and tender gynaecomastia, very scant body hair, and reduced testicular size. Endocrine investigations revealed severe hypogonadism, with a serum testosterone of $0.8 \mathrm{nmol} / 1$ (normal range 10.0-30.0), and serum FSH and LH both undetectable. Prolactin level, and magnetic resonance imaging of the pituitary and hypothalamus were normal. Dynamic pituitary function testing with glucagon, thyrotropin-releasing hormone (TRH) and LH-releasing hormone showed normal responses of cortisol, growth hormone (GH), thyrotropin (TSH), and FSH/LH. A diagnosis of severe hypogonadotrophic hypogonadism due to anabolic steroid abuse was made.

$\mathrm{He}$ initially refused to give up anabolic steroids and was therefore offered Sustanon treatment with a contract that he in return would stop other steroids. It was hoped that this might at least act as a 'damage limitation' strategy short-term. Sustanon $250 \mathrm{mg}$ intramuscularly (im) was given every two weeks, and serum testosterone levels rose into the normal range (see figure). His libido, potency and hair growth returned to normal. His cessation of anabolic steroid misuse was supported by urine screening tests. After 15 months on Sustanon, he had given up body-building and was in fulltime education. He voluntarily stopped treatment, but serum testosterone fell from 14.0 to $8.5 \mathrm{nmol} / 1$ (see figure) and impotence rapidly returned. To stimulate testicular function he was given injections of HCG over the next three months (10 000 units im weekly for one month, 5000 units weekly for one month, and 2500 units for one month). Within a week of starting treatment, libido had greatly improved, and spontaneous nocturnal ejaculations occurred. Serum testosterone levels and potency returned to normal over the three months of treatment (see figure). In 30 months of subsequent follow-up, the patient remained clinically and biochemically eugonadal and symptom-free on no treatment.
Endocrine Unit, Liverpool L9 1AE, UK GV Gill

Accepted 28 May 1997 


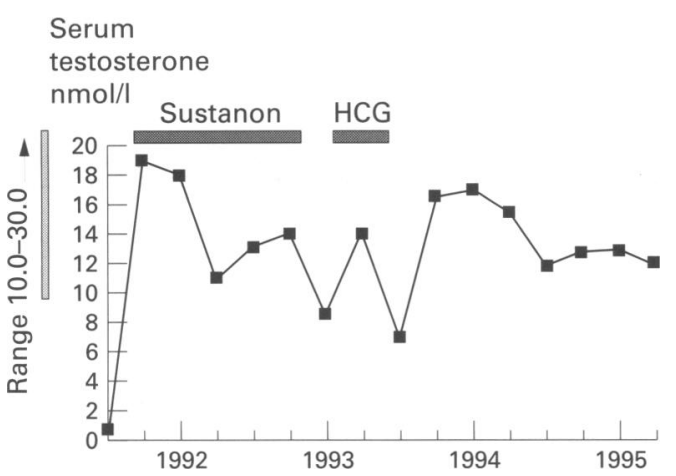

Figure Serum testosterone levels during Sustanon and then HCG treatment for anabolic steroid-induced hypogonadotrophic hypogonadism

\section{Discussion}

Anabolic steroid abuse amongst athletes is widespread, though potentially hazardous. ${ }^{2-4}$ The extent of use is difficult to ascertain, but McKillop ${ }^{6}$ recorded that eight out of $41(20 \%)$ Scottish body-builders were using various anabolic preparations, and Buckley and colleagues in the $\mathrm{USA}^{7}$ recorded $7 \%$ of male senior (over 16 years) school children as misusing anabolic drugs. A variety of preparations are in use, but doses are often high and multiple regimes (including both oral and injectable preparations) are often used. ${ }^{5}$ Critical examination of the effect of anabolic steroids in enhancing performance has given inconsistent results, ${ }^{15}$ though in the field of body-building they certainly improve physique.

Adverse effects include acne, gynaecomastia, reduced libido, testicular atrophy, impotence and azoospermia. Liver dysfunction (with possible long-term hepatoma risk), and adverse lipid profiles also may occur. ${ }^{15}$ There are wellrecorded neuropsychiatric effects, including mood swings, cognitive impairment and occasionally mania. ${ }^{8}$ The hypogonadal side-effects are due to $\mathrm{FSH} / \mathrm{LH}$ inhibition and even after stopping the anabolic drugs, hypogonadotrophic hypogonadism may persist for many months, or even longer. There is very little recorded information on the use of HCG in this situation. Martikainen and colleagues ${ }^{9}$ tested the effect of a single HCG dose (50

1 Bagatell CJ, Bremner WJ. Androgens in men - users and abusers. N Engl f Med 1996;334:707-14.

2 Lamb DR. Anabolic steroids in athletics: how well do they work and how dangerous are they? Am $\mathcal{f}$ Sports Med 1984; work and

3 Perry HM, Littlepage BNC. Misusing anabolic drugs. $B M F$ 1992;305:1241-2.

4 Wilson JD. Androgen abuse by athletes. Endocr Rev 1988;9: 181-99.

5 Wu FCW. Testicular steroidogenesis and androgen use and abuse. Clin Endocr Metab 1992;6:373-403.

6 McKillop G. Drug abuse in bodybuilders in the west of Scotland. Scot Med $\mathscr{f}$ 1987;32:39-41.

7 Buckley WE, Yesalis CE, Friedl KWE, Anderson WA, Streit $\mathrm{AL}$, Wright JE. Estimated prevalence of anabolic steroid use among male high school seniors. $尹 A M A$ 1988;260: 3441-5.

\section{Learning points}

- abuse of anabolic steroids by athletes and body-builders is common

- prolonged anabolic steroid misuse may lead to hypogonadism due to FSH/LH suppression

- anabolic steroid-induced hypogonadism may be severe and prolonged; treatment with HCG may be helpful

units/kg im) in six anabolic-using power athletes. They found an attenuated acute testosterone rise (similar to that seen in the pre-pubertal state), and suggested that FSH/LH priming may be necessary for an adequate response. Jarrow and Lipshultz ${ }^{10}$ reported two cases of hypogonadotrophic hypogonadism (one a body-builder and the other a weight-lifter), and in one case treatment with HCG (no details of the regime used were given) restored potency and fertility. The dose used in the case reported here was therefore chosen arbitrarily, but appeared effective.

This case also demonstrates the prolonged nature of anabolic-induced hypogonadism, even after drug cessation. It also introduces the perhaps controversial option of offering Sustanon treatment as a 'damage-limitation' procedure analogous to opiate drug withdrawal programmes. There was additionally a good response to HCG, even after prolonged suppression of the hypothalamo-pituitarygonadal axis. During therapy, FSH/LH levels were not measured and presumably remained at least initially suppressed. The maintenance of clinical and biochemical eugonadism after HCG was stopped, however, indicates normal function of the pituitary-testicular axis, in a manner similar to that seen with HCG treatment of delayed puberty. It is not entirely possible to exclude coincidental spontaneous recovery of $\mathrm{FSH} / \mathrm{LH}$ release. Such recovery from anabolic steroid induced hypogonadism can take several months or more, ${ }^{4}$ though there is little definite information in the literature on this. However, the prompt improvement after initiating HCG treatment strongly suggests a genuine effect.

8 Tung-Ping S, Pagliano M, Schmidt PJ, Pickar D, Wolkowitz $\mathrm{O}$, Rubinow DR. Neuropsychiatric effects of anabolic steroids in male normal volunteers. $¥ A M A$ 1993;269:2760-4.

9 Martikainen H, Alen M, Rahkila P, Vihko R. Testicular responsiveness to human chorionic gonadotrophin during transient hypogonadotrophic hypogonadism induced by androgenic/anabolic steroids in power athletes. F Steroid Biochem 1986;25:109-12.

10 Jarrow JP, Lipshultz LI. Anabolic steroid induced hypogonadotrophic hypogonadism. Am $\mathcal{F}$ Sports Med 1990;18:429-31. 